\title{
EXTRACTION OF ROAD FROM SATELLITE RESOLUTION IMAGES USING MATLAB
}

\author{
V. Leela Satyanarayana \\ Assistant Professor \\ Department of Electronics and Communication Engineering \\ Potti Sriramulu Chalavadi Mallikharjuna Rao College of Engineering \& Technology \\ Ch.Gopi Chandana, K.Bindusha, D.Padmanabhudu, G. Shahanaaz bhanu \\ Students \\ Department of Electronics and Communication Engineering \\ Potti Sriramulu Chalavadi Mallikharjuna Rao College of Engineering \& Technology \\ Vijaywada, India.
}

\begin{abstract}
The importance of road extraction from satellite images arises from the fact that it greatly enhances the efficiency of map generation and it provide huge volume of data and monitor events and areas without any interference. This has motivated, dedicated and focused on the development of efficient and advanced methods for object detection and extraction from satellite images. Roads are important bowed object as they are used in urban planning, emergency response, route planning etc. Automatic road detection from satellite images has now become an important topic and roads play a vital role in urban planning and thus its extraction can be great helpful for human expertise. In this paper, to enhance the image and to strengthen the road feature with the surrounding environment color gradient we use some methods for road extraction from satellite images based on the concept histogram equalization, Otsu's method of image segmentation, median filter and edge detection operations of image processing. The experiment result of this paper is to discover the potential of high resolution satellite images for detecting and extracting the road network in a robust manner.
\end{abstract}

KEYWORDS: Road extraction, Otsu's method, Image segmentation, morphological operations.

\section{INTORDUCTION:}

Satellite images obtain information of areas which are difficult to reach and provide monitor event, huge volume of data from GIS database. For transportation, city planning and urban planning, roads are very essential linear features. Automatic road detection from satellite images has now become an important topic in photogrammtery and it is also helpful in urban planning. The artificial features, mainly including bridges, vehicles and roads etc. are identified and extraction of buildings and roads accounted for 80 percent high resolution satellite images.

Satellite images often contain noise and several objects. Therefore, these images are preprocessed and enhanced before the extraction of objects and to reduce the noise in the image by using filters. Median filter is one of the best filters that reduce the noise in the image. Automatic object detection with the help of computers was developed but these object detection algorithms localize objects and annotate them with an object class label. Both the tasks are very difficult that on one hand, the detection method must be robust for different images and request account for changes in the object. The object in the images of any size and at anywhere. The objects in the satellite images are dividing into three categories of point, line and area type. It can also be used in transportation database, city planning, urban planning, military field and map updating.

\section{LITERATURE SURVEY:}

Many researches have been carried out in automatic methods for road detection from satellite images. lot of methods and algorithms are introduced to extract road from satellite images. At present, many researchers from various aspects put forward various research methods and algorithms that 
International Journal of Engineering Applied Sciences and Technology, 2020 Vol. 4, Issue 12, ISSN No. 2455-2143, Pages 708-714

Published Online April 2020 in IJEAST (http://www.ijeast.com)

resulting in a many number of practical results. Now, forming methods, Huang et al (2012) have given a multi -stage method to extract road from high resolution satellite. This paper presents a multi stage method, consisting of automatic information extraction and semi-automatic post-processing in extracting road information from high resolution satellite images. In this stage 1 is automatic information this consists of three modules: the MsE, SRG and skeletonization. Jose Hormese, Dr.c.Saravanan (2015) has introduced Vectorization . The work manages extraction of roads from satellite pictures. Gruen et al (1997) have given a technique that included dynamic programming. Keaton et al (2002) proposed a technique for street extraction in which a seed point is chosen from the street and afterward a level set technique is utilized to develop the area. Iterative smoothing is performed which further improved the removed segment and afterward street centerline is evaluated. He et al. (2004) built up a street zone identification which has two modules. In first module, scene zone was decreased for the calculation of mean and fluctuation of Gaussian circulation. In second module the street zone was removed and afterward limits that fit the consequence of street were strengthened. Fluffy strategy was applied for street extraction in a paper (Mohammadzadeh et al., 2009, 2007). Hu et al. (2009) has embraced Least Squares Template Matching (LSTM) for fitting the straight items with a Scalable Slope Edge (SSE) model. A strategy was displayed for direct element extraction which has incorporated the SSE with a LSTM model for handling uproarious pictures and obscured edges. $\mathrm{Li}$ et al (2009) gave a calculation in which edge separating was performed to remove streets. Stage division, gathering and advancement were then applied for streets that are typically harder to recognize due to clamor and furthermore to fill enormous holes. Wu et al (2009) have proposed a calculation for unstructured street recognition. Right now, channel is applied on unique pictures to stifle the stochastic kind of commotion and afterward multithreshold is chosen by Otsu strategy based on two-top strategy. At long last, the essential edge is recognized with the assistance of watchful administrator and tasks of arithmetic morphology are utilized, which brings about clear street picture.

\section{DATA FLOW}

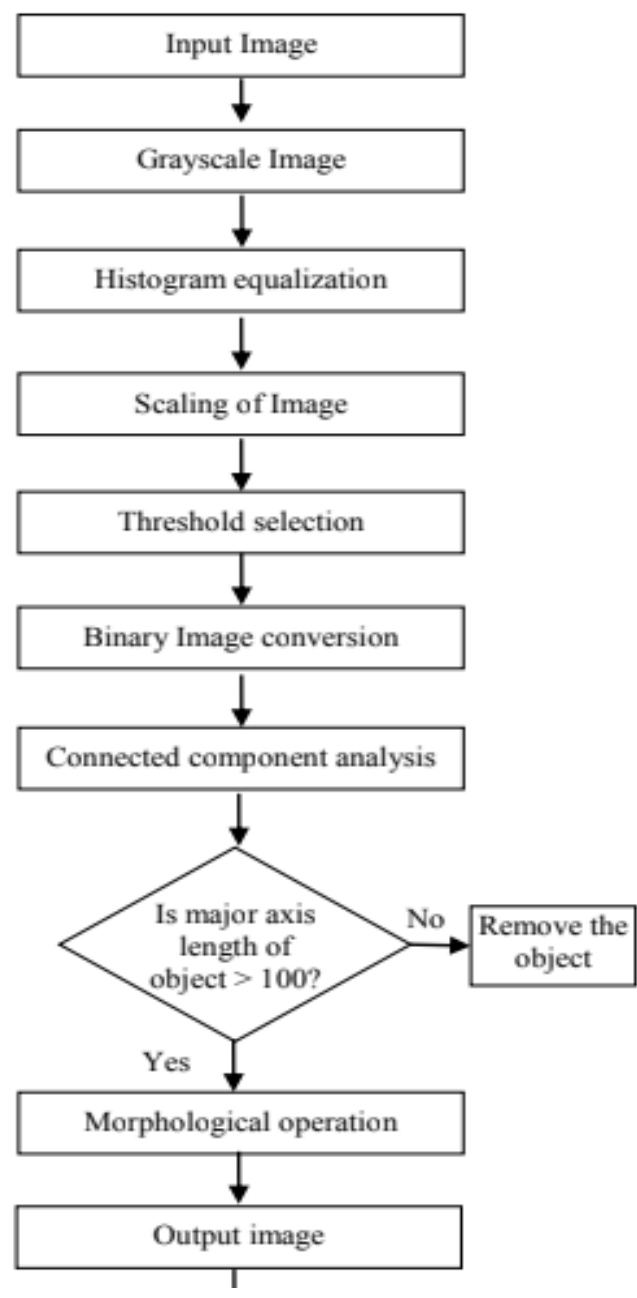

Fig.1: Road Extraction Methodology.

\section{DESCRIPTION OF DATA FLOW:}

The image is extracted from the satellite images by using GIS data base. In this method, the input image is converted to grayscale image. Therefore the color image is converted to grayscale image. Histogram equalization is a strategy which remaps input picture pixels with the goal and then we apply the threshold selection by this we can easily obtain the binary image. Median filter is used only to reduce the noise in the image but the unwanted objects in the images are removed by using morphological operation and then we get output of the image in that we detect the road network and extract the road network in the image 
International Journal of Engineering Applied Sciences and Technology, 2020

Vol. 4, Issue 12, ISSN No. 2455-2143, Pages 708-714

Published Online April 2020 in IJEAST (http://www.ijeast.com)

\section{BLOCK DIAGRAM}

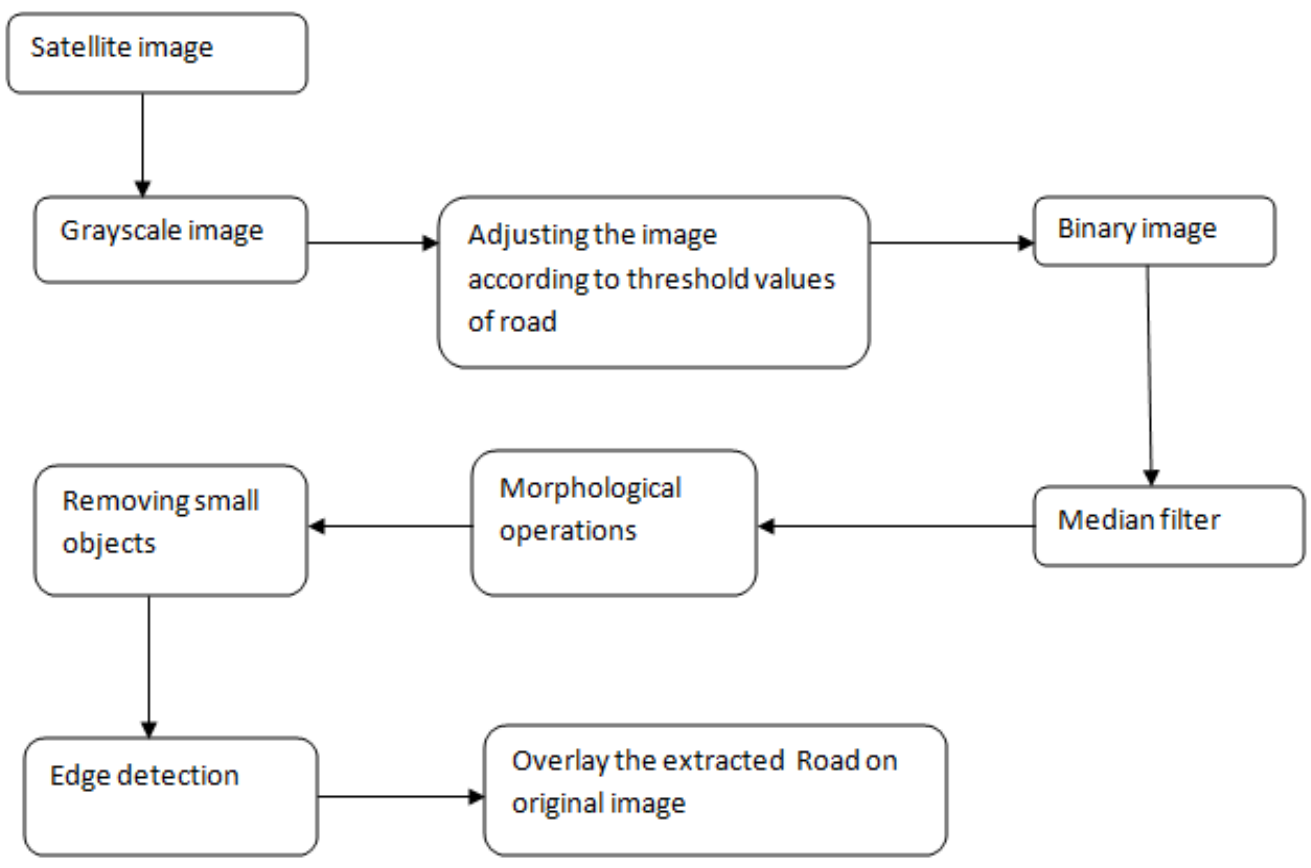

\section{DESCRIPTION OF BLOCK DIAGRAM}

As the high resolution satellite images have become easily available in GIS database, we extract the images from the satellite images by using GIS database. The extracted image consists of noise and blur in the image, to avoid this problem we use some image processing techniques to get original image.

\section{A. Gray scale transformation:}

The colored image consists of three independent colors: red, green \& blue. The color image increases complexity of image and also increases the processing time. Grayscale pictures are single channel pictures on which differentiate, shape; edges can be examined without utilizing color channels. In this way the color pictures are changed over into gray scale images.

\section{B. Histogram equalization:}

Histogram equalization is a technique which reconstructs the input image pixels by this histogram may be achieved and it is very helpful in enhancing the contrast of the image. This histogram equalization applied on the gray scale image.

\section{Binary image transformation:}

\section{Adjustment of threshold:}

The given input image is converted to a gray image and then the gray scale is adjusted to the threshold values of the road. The obtained gray scale image is converted to binary image by adjusting the threshold value. Initially threshold range for road is about 0.5 to 0.9 to remove the unwanted stuff. By adjusting the threshold value we can easily get the binary image.

\section{Binarization:}

After threshold selection, image is converted to binary image where in the image we consists of different pixel values in that all pixel value which are greater than the threshold are replaced by 1 and the remaining pixel values are replaced by 0 . In this way we get result as a black \& white image.

\section{Median filterization:}

The image consists of some noise in the image to overcome this problem we use filters. Median filter is one of the most useful filters while compared to the entire filter. This median filter reduces the noise in the binary image and gives noiseless image as output. 


\section{International Journal of Engineering Applied Sciences and Technology, 2020 Vol. 4, Issue 12, ISSN No. 2455-2143, Pages 708-714 \\ Published Online April 2020 in IJEAST (http://www.ijeast.com)}

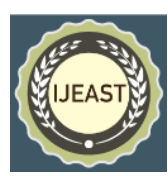

\section{E. Morphological Operation:}

The extracted roads from the image still contain some hole \& noises. The problem in this is the connected component sometimes cannot identify small ground objects like building, lanes, vehicles etc. to improve accuracy of result we use some operations of mathematical morphology like opening, closing, dilation \& erosion. Matheron \& serre has introduced method of mathematical morphology in 1964. The application of morphology is an approaching which objects features are identified through their shape.

The area opening operation removes those connected components which have less number of pixels. Thinning operation thins the connected components to lines and pixels are removed in such manner that an object which does not contains any holes reduces to minimal. Opening operation is used for both erosion \& dilation.

\section{F. Edge Detection:}

Edge detection incorporates an assortment of numerical techniques that target distinguishing focuses in an advanced image at which the image splendor changes strongly or, all the more officially, has discontinuities. The focuses at which image brilliance changes strongly are ordinarily sorted out into a lot of bended line sections named edges. A similar issue of discovering discontinuities in onedimensional signs is known as step identification and the issue of discovering signal discontinuities after some time is known as change discovery. Edge location is a crucial apparatus in image handling,

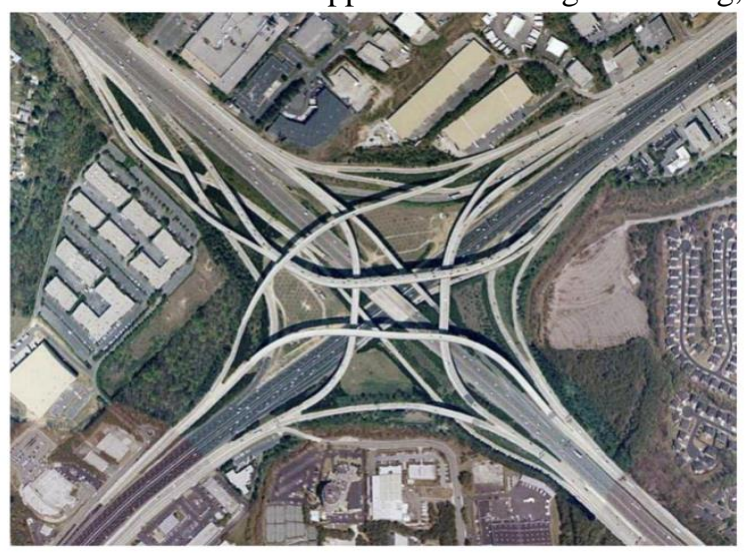

Fig. 2: Original Image. machine vision and PC vision, especially in the territories of highlight discovery and highlight extraction.

\section{GRAPHICAL REPRESENTATION:}

Graphical interface is formed in MATLAB. It contains a figure window during which menus, content, designs, and catches then forth are given. To open GUI, select File/New/GUI from menu or type "control" on Command Window and choose "Make New GUI". Design Editor is then opened during which the presence of GUI is often planned. On the left of the Layout Editor a couple of catches are given for embeddings objects like switch catches, push catches, static content, tomahawks box then on. GUI is then made with the assistance of Layout Editor. Property Inspector is employed to switch the thing properties like content text style, shading then forth.

For sparing a GUI, click Save As from File menu and sort the name of document with none expansion (Hunt et al., 2012). after sparing it, two sorts of documents are made double record with '.fig' expansion and a M-record with '.m' expansion. At the purpose when the M-document is run from instruments menu, GUI shows up with chosen catches and menus of format editorial manager. To relegate the usefulness to the GUI catches callback work is employed. Directions are added to the catches within the wake of opening the M-document. Presently it's spared and executed. GUI window is at that time opened and on tapping the catches wanted activity are performed.

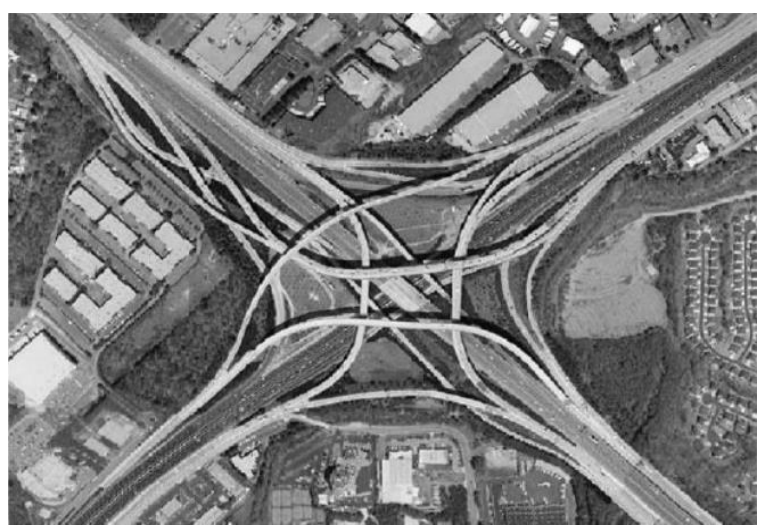

Fig. 3: Gray Scale Image. 
International Journal of Engineering Applied Sciences and Technology, 2020 Vol. 4, Issue 12, ISSN No. 2455-2143, Pages 708-714

Published Online April 2020 in IJEAST (http://www.ijeast.com)

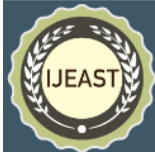

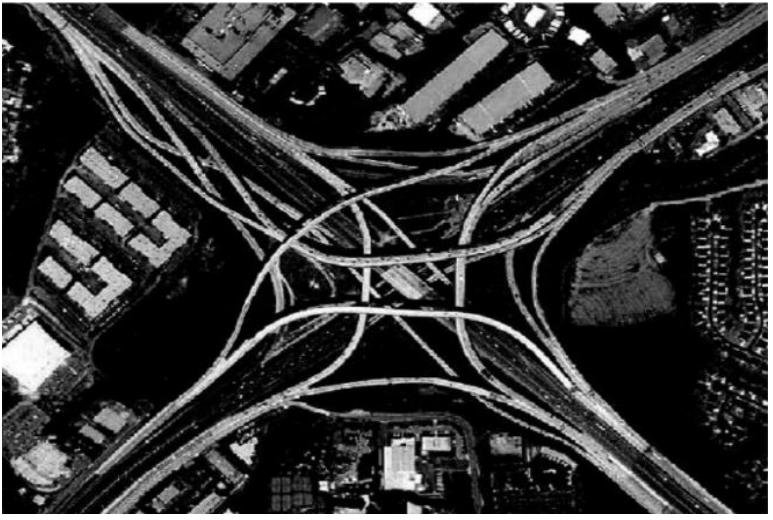

Fig. 4: Image Obtained After Adjusting Threshold.

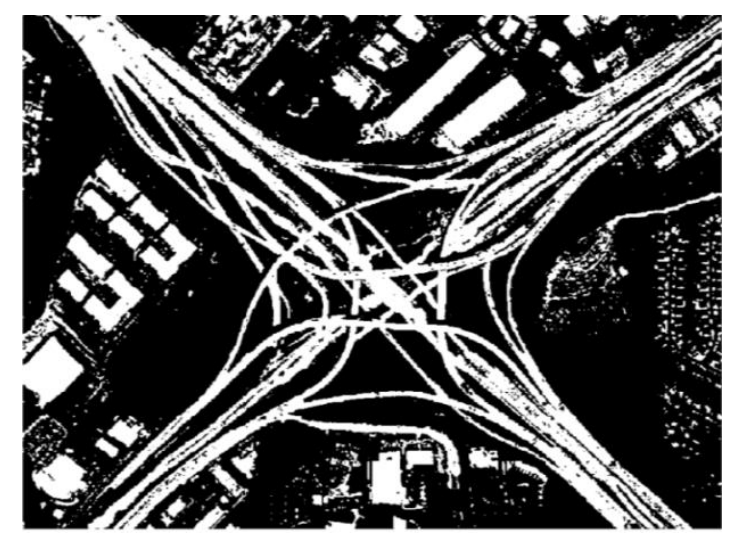

Fig. 5: Binary Image.

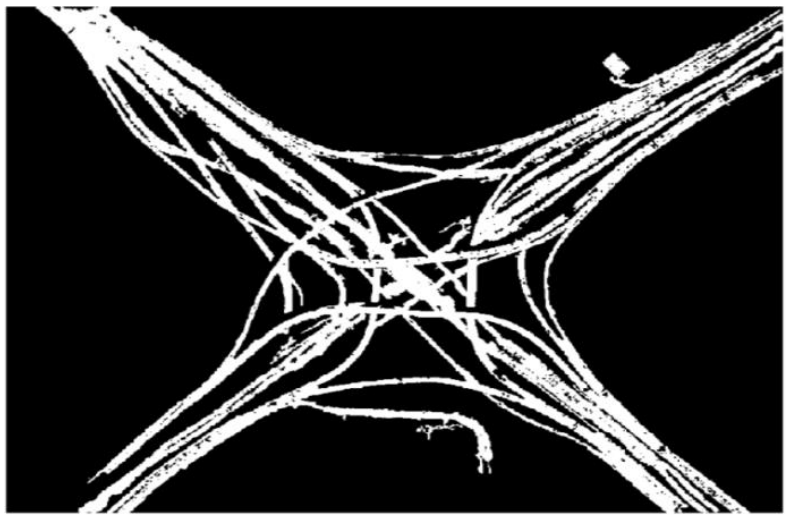

Fig. 6 :Median Filtered Image.

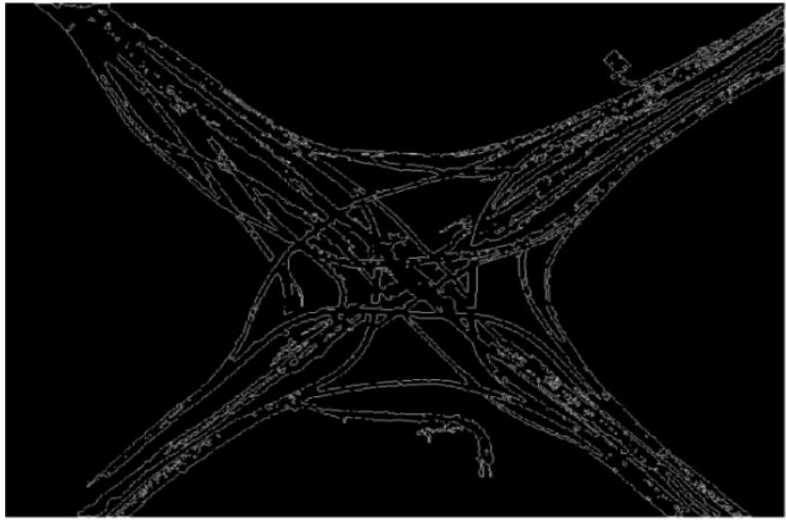

Fig.7: Morphological Filtered Image.

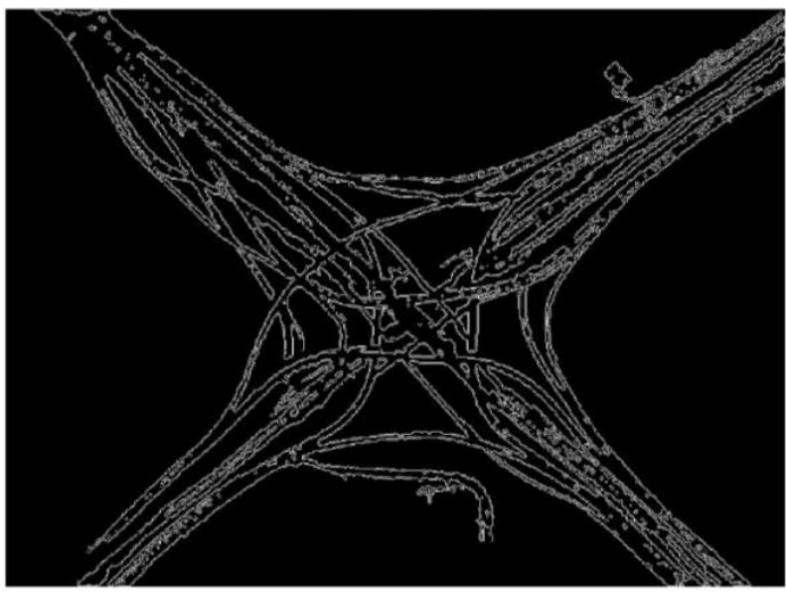

Fig.8 :Edge Detection.

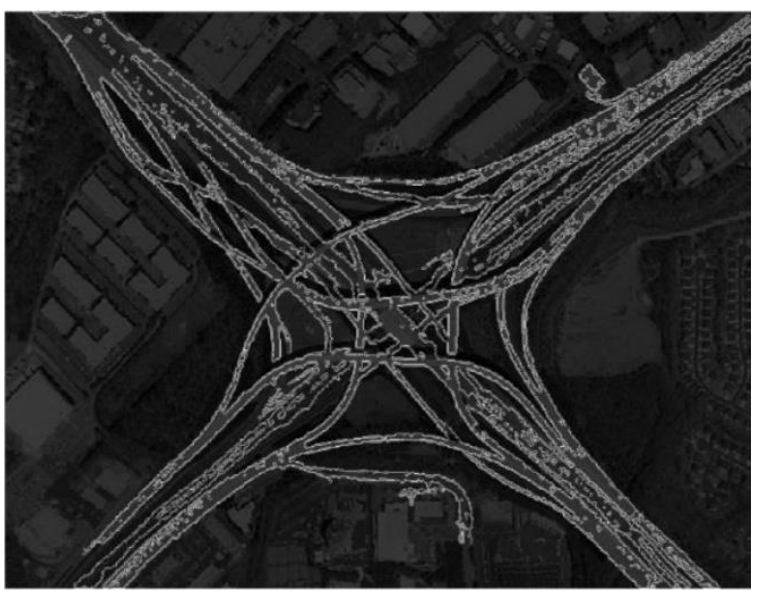

Fig.9: Overlay of Gray Scale Image. 


\section{International Journal of Engineering Applied Sciences and Technology, 2020 Vol. 4, Issue 12, ISSN No. 2455-2143, Pages 708-714 \\ Published Online April 2020 in IJEAST (http://www.ijeast.com)}

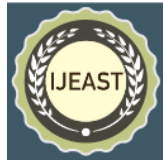

\section{RESULT}

The aftereffects of proposed strategy for street ID and extraction are appeared in above figures. The proposed strategy has been applied on high goals satellite pictures. Figure 2 shows the primary shaded picture. This picture is of Washington DC caught by WorldView-3 on April 18, 2015. It's downloaded from Digital Globe site. Figure 4 shows the binary image after adjusting the threshold by using Otsu's method. From the median filtered image small objects are removed which are unnecessary and whose pixels are less than 60 using 'bwareaopen'. This is shown in the Figure 5. The image still contains many unwanted pixels. One of easiest way to eliminate unwanted objects from a picture is by applying morphological operations. Morphological operations are those operations wont to remove undesired pixels supported the foreground and therefore the background of a picture. Since the operations are done on the binary image, the MATLAB function used is 'bwmorph ()' and 'remove' operation. The image obtained after applying morphological operations is given in Figure 6. After applying the morphological operations we get the clean roads but it's vital to get the sides of those roads for clear identification of the roads. Gradient filter is used for the sting detection and therefore the sort of operator used for the detection is 'sobel'. Sobel operator is employed because the edges are extracted with greater accuracy. The edge detection is shown in the Figure 7. The final step is to overlay the extracted road onto the scalar image of the first image. Overlaying of the result helps for instance the accuracy of the road extraction. In the final image, the skinny lines indicate the paths of roads within the image. The ultimate image is given in Figure 8.

\section{CONCLUSION}

Right now, identification and extraction technique has been proposed and actualized for top goals satellite pictures. This strategy depends on Otsu's division technique, associated part investigation and morphological tasks. In proposed strategy, high goals satellite picture is modified over to grayscale picture, rescaling of picture were performed and afterward complexity of picture was improved by histogram leveling. A edge was chosen by utilizing Otsu's strategy and utilizing this edge, picture was changed over into double picture. From that time forward, associated segment examination was performed which evacuated the territories which weren't related to street organize. From that time forward, morphological tasks were utilized to refine the image.

At that time, separated street organize has been found. After street location, a grayscale picture with red hued street organize was found. There are a couple of constraints immediately. The territories related to street system couldn't been expelled. To bargain with this constraint, on the off chance that width of articles might be determined, at that time this constraint might be evacuated by applying a foothold on width of things.

\section{REFERENCES}

Alemu, M.M., 2016. Automated Farm Field Delineation and Crop Row Detection from Satellite Images. Geo-Information Science and Earth Observation of the University of Twente.

Alian, S., 2007. Identifying Curviness of Overpass Mountain Roads from Remote Sensing Data. International Institute For Geo-Information Science And Earth Observation.

Zhang, H., Lin, H., Li, Y., Zhang, Y., 2013. Feature Extraction for High-Resolution Imagery Based on Human Visual Perception. International Journal of Remote Sensing 34, 1146- 1163. https://doi.org/10.1080/01431161.2012.718459

Wang, M., Zhang, S., 2011. Road Extraction from High-Spatial-Resolution Remotely Sensed Imagery by Combining Multi-Profile Analysis and Extended Snakes Model. International Journal of Remote Sensing 32, 6349-6365. https://doi.org/10.1080/01431161.2010.508801

Huang, Z., Jia, X., 2012. Integrating Remotely Sensed Data, GIS and Expert Knowledge To Update Object-Based Land use/land Cover Information. International Journal of Remote Sensing 33, 905921. https://doi.org/10.1080/01431161.2010 .536182

Gruen, A., Li, H., 1997. Semi-Automatic Linear Feature Extraction by Dynamic Programming and LSB-Snakes. Photogrammetric Engineering and Remote Sensing 63, 985-995.

Keaton, T., Brokish, J., 2002. A Level Set Method for the Extraction of Roads from Multispectral Imagery, in: Applied Imagery Pattern Recognition 


\section{International Journal of Engineering Applied Sciences and Technology, 2020 Vol. 4, Issue 12, ISSN No. 2455-2143, Pages 708-714 \\ Published Online April 2020 in IJEAST (http://www.ijeast.com)}

Workshop. Washington DC, pp. 141-147. https://doi.org/10.1109/AIPR.2002.1182268

Wang, S., Liu, C., Wu, S., Qianqian, N., Wang, Y., Shia, Z., Haifeng, Z., 2012. Automatic Extraction of Foreground Objects from Mars Images. Geo-spatial Information Science 15, 17-25. https://doi.org/10.1080/10095020.2012.708147

Wu, W., ShuFeng, G., 2009. Research on Unstructured Road Detection Algorithm Based on the Machine Vision, in: Asia-Pacific Conference on Information Processing. IEEE, Shenzhen, pp. 112115. https://doi.org/10.1109/APCIP.2009.164

Mohammadzadeh, A., ValadanZoej, M.J., Tavakoli, A., 2007. Automatic Class Mean Calculation of Road Surface From Ikonos Images Using Fuzzy Logic and Particle Swarm Optimization, in: Photogrammetric Image Analysis. ISPRS, Munich, pp. 119-124.

Mohammadzadeh, A., Zoej, M.J.V., Tavakoli, A., 2009. Automatic Main Road Extraction from High Resolution Satellite Imageries by Means of Particle Swarm Optimization Applied to a Fuzzy-Based Mean Calculation Approach. Journal of the Indian Society of Remote Sensing 37, 173-184. https://doi.org/10.1007/s12524-009-0021-y

Li, Y., Briggs, R., 2009. Automatic Extraction of Roads from High Resolution Aerial and Satellite Images with Heavy Noise, in: World Academy of Science, Engineering and Technology. pp. 416-422.

Lin, X., Liu, Z., Zhang, J., Shen, J., 2009. Combining Multiple Algorithms for Road Network Tracking from Multiple Source Remotely Sensed Imagery: A Practical System and Performance Evaluation. Sensors 9, 1237-1258. https://doi.org/10.3390/ s90201237

Soni, A., Pandey, N., Halarnkar, P., 2014. Review on Image Object Extraction. International Journal of Current Engineering and Technology 4, 864-869.

Sujatha, C., Selvathi, D., 2015. Connected Component-Based Technique for Automatic Extraction of Road Centerline in High Resolution Satellite Images. Eurasip Journal on Image and Video Processing 8, 1-16. https://doi.org/10.1186/s13640015-0062-9
Sundaram, M., Ramar, K., Arumugam, N., Prabin, G., 2011. Histogram Modified Local Contrast Enhancement for Mammogram Images. Applied Soft Computing Journal 11, 5809-5816. https://doi.org/10.1016/j.asoc.2011.05.003

Tiwari, D., Saroha, G.P., Bhushan, U., 2012. Road Network Extraction from Satellite Images by Active Contour (Snake) Model and Fuzzy C-Means. International Journal of Advanced Research in Computer Science and Software Engineering 2, 195198. 